\title{
Percepción de la imagen social del profesional de enfermería, Chachapoyas, región Amazonas, 2020
}

\section{Perception of the social image of the nursing professional, Chachapoyas, Amazonas region, 2020}

\author{
Jordany Siday Lobato Diaz', Yshoner Antonio Silva Diaz ${ }^{2}$
}

\begin{abstract}
RESUMEN
El objetivo del estudio fue determinar la percepción de la imagen social del profesional de enfermería en pacientes del puesto de salud Pedro Castro Alva, en el distrito de Chachapoyas, región Amazonas, Perú, 2020. El enfoque fue cuantitativo, de nivel descriptivo, de tipo prospectivo, observacional, transversal y univariado. La muestra fue de 49 pacientes; se recolectó los datos de forma virtual aplicando el cuestionario "Imagen social del profesional de enfermería". Los resultados fueron, que del $100 \%$ de pacientes el $54 \%$ tienen una percepción de la imagen social del profesional de enfermería favorable y el $46 \%$ desfavorable. En las dimensiones, información el $73.5 \%$ tiene una percepción desfavorable y el $26.5 \%$ favorable, en el campo de presentación el $71.4 \%$ posee una percepción favorable y el $28.6 \%$ desfavorable, en la actitud el $75.5 \%$ tiene una percepción favorable y el $24.5 \%$ desfavorable y en la dimensión religión el $69.4 \%$ posee una percepción favorable y el $30.6 \%$ desfavorable. Se concluye que gran parte de los pacientes tiene una percepción favorable acerca de la imagen social del profesional de enfermería, y en la dimensión información es desfavorable.
\end{abstract}

Palabras clave: Imagen social, profesional de enfermería

\begin{abstract}
The objective of the study was to determine the perception of the social image of the nursing professional in patients of the Pedro Castro Alva health post, in the Chachapoyas distritic, Amazonas región, Peru, 2020. The approach was quantitative, descriptive, prospective, observational, cross-sectional and univariate. The sample consisted of 49 patients; Data was collected virtually by applying the questionnaire "Social image of the nursing professional". The results were: of $100 \%$ of patients, $54 \%$ have a favorable perception of the nursing professional's social image and $46 \%$ unfavorable. In the dimensions: information $73.5 \%$ have an unfavorable perception and $26.5 \%$ favorable, in the field of presentation $71.4 \%$ have a favorable perception and $28.6 \%$ unfavorable, in attitude $75.5 \%$ a favorable perception and $24.5 \%$ unfavorable and in the religion dimension, $69.4 \%$ have a favorable perception and $30.6 \%$ unfavorable. It is concluded that a large part of the patients has a favorable perception about the social image of the nursing professional, and in the Information dimension it is unfavorable.
\end{abstract}

Keywords: Social image, nursing professional

'Bachiller en Enfermería de la Facultad de Ciencias de la Salud de la Universidad Nacional Toribio Rodríguez de Mendoza de Amazonas. Correo electrónico: jodanylobato@gmail.com

${ }^{2}$ Docente adscrito a la Facultad de Ciencias de la Salud de la Universidad Nacional Toribio Rodríguez de Mendoza de Amazonas. Doctor en

Ciencias de la Educación. Correo electrónico: yshoner.silva@untrm.edu.pe. 


\section{INTRODUCCIÓN}

El cuidado siempre ha estado presente desde épocas muy antiguas, el ser humano se reunía en grupos para así poder prolongar su supervivencia y con ello cuidarse los unos con los otros, es así que este cuidado paso por diversos periodos de evolución y distintas definiciones, esto pone en evidencia que debido al hecho histórico la mujer por elección se le relacionada al cuidado, y actualmente esto también tiene influencia en la familia. (Costana, 2014, p.2)

Se define a enfermería de manera general como una ciencia que se encarga del cuidado de heridos y enfermeros y tareas de asistencia sanitaria bajo las prescripciones de un médico, con ello poniendo un concepto limitado de los que realmente es esta profesión y demostrando a la población la escaza información que existe. (RAE,2020 p.1)

A pesar que dicha profesión ha cambiado rotundamente siempre ha estado ligada de creencias basadas en su pasado, tal vez debido que gran parte de las personas crean en sus mentes impresiones distorsionadas. Actualmente la formación que se ejerce en el ámbito universitario es poco conocida por la mayoría de la población como menciona Heirle que a pesar que las enfermeras (os) tiene logros espectaculares, estos no han cambiado la imagen que de ellos se transmiten. (Heirle,2009,p.4)

Por otro lado, otra de las percepciones erróneas que se ha visto ligada, es con la aparición del modelo biomédico en la cual estos, con el significado de la curación se cumplía las ordenes de la prescripción médica, es por ello que gran parte de la población ven al profesional como ayudante del médico y con ende también no se transmite la imagen autónoma ya que gran parte de las decisiones que son tomadas, es para el correcto cuidado de estos. (Errasti B., Arantzamendi M., Canga N. 2012, p.12).

En América, existe deficiencias en cuanto al número de profesionales de enfermería, su distribución para la disposición en diferentes países y disminución de la presencia masculina, es por ello conveniente aumentar el trabajo en zonas rurales y de difícil acceso e invertir en formación y empleo. Además de ello con la llegada de la pandemia del Covid- 19 se ha demostrado que dicha profesión siempre estuvo en primera línea de atención y cuidado recalcando la necesidad e importancia de los profesionales de la salud para suplir las demandas y que estos mismos trabajen en las mejores condiciones. (OMS,2020, p.2)

Asimismo, en Latinoamérica realizaron un estudio cuantitativo descriptivo sobre percepción de la imagen social del rol de enfermería cuyos los resultados enmarcaron déficit de percepción $(79,70 \%)$ sobre las capacidades y habilidades que desempeña el profesional de enfermería en su ámbito laboral por lo que los autores concluyeron que existe la necesidad de promover el verdadero rol del profesional de enfermería, con el propósito de cambiar la percepción, la imagen distorsionada e incompleta que la sociedad tiene. (Mieles \& Parreño,2017,p.14)

Cabe recalcar que los medios de comunicación son una de las causas por el que el perfil profesional no se ve reflejada adecuadamente, lo cual no ayuda a proyectar las habilidades que poseen y el trabajo que realizan, exponiendo a través del cine y la televisión una representación negativa en las cuales se le muestra enfermera religiosa, ayudante del médico, enfermera heroína y militar entre otros. (Muñoz, 2007, p.10).

En el Perú se investigó sobre la imagen social del profesional de Enfermería según la percepción de los pacientes del Hospital Huaycán, se encontró que, el $55,4 \%$ de los pacientes poseen una buena imagen social del profesional de Enfermería, a comparación de que $44,4 \%$ distingue de forma regular y el $0,2 \%$ malo. Los investigadores concluyeron que gran parte de pacientes hospitalizados que tuvieron interrelación enfermero paciente poseen una adecuada apreciación del profesional en el Hospital Huaycan. (Mauricio, C., \& Janjachi, B,2017,p.13).

Se pude evidenciar que la imagen de la enfermera esta distorsionada, no concuerda con los modelos que se promueven en las universidades, mucho menos contrasta con las funciones que desarrolla; las personas refieren que la enfermera se dedica a colocar inyectables, medir la presión arterial, ayudar al médico, etc, confundiéndose muchas veces con la labor del técnico de enfermería, sin embargo, la profesión ha evolucionado en los últimos años, logrando importantes avances, pasando de un modelo de cuidado practico a uno fundamentado en modelos teóricos.

\section{MATERIAL Y MÉTODO}

El estudio fue de enfoque cuantitativo; enmarcado en el nivel descriptivo. Por el tipo de intervención fue observacional; según la planificación de la toma de datos fue prospectivo; según el número de ocasiones en que se midió la variable fue transversal y según el número de variables de interés fue univariado. (Supo, José. 2015.p.2).

La muestra fue de 49 pacientes que acuden al Puesto de salud Pedro Castro Alva; se recolectó los datos de forma virtual aplicando el cuestionario "Imagen social del profesional de enfermería". 


\section{RESULTADOS}

Tabla 1

Percepción de la Imagen Social del profesional de enfermería.

\begin{tabular}{lcc}
\hline $\begin{array}{l}\text { Percepción de la imagen } \\
\text { Social del enfermero }\end{array}$ & fi & $\mathbf{\%}$ \\
\hline Favorable & 26 & 54.0 \\
Desfavorable & 23 & 46.0 \\
\hline Total & $\mathbf{4 9}$ & $\mathbf{1 0 0}$ \\
\hline
\end{tabular}

En la tabla 1, se observa que del 100\% (49) de los pacientes, el 54\% (26) poseen una percepción favorable de la imagen social del profesional de enfermería y el 46\% (23) desfavorable.

\section{Tabla 2}

Percepción de la Imagen Social del profesional de Enfermería de acuerdo a dimesiones.

\begin{tabular}{|c|c|c|c|c|}
\hline \multirow{2}{*}{$\begin{array}{l}\text { Percepci } \\
\text { ón de la } \\
\text { Imagen } \\
\text { Social }\end{array}$} & \multicolumn{4}{|c|}{ Dimensiones } \\
\hline & $\begin{array}{l}\text { Informa } \\
\text { ción }\end{array}$ & $\begin{array}{l}\text { Campo de } \\
\text { presentación }\end{array}$ & Actitud & Religión \\
\hline & fi $\%$ & $\%$ & fi $\%$ & $\%$ \\
\hline $\begin{array}{l}\text { Favorab } \\
\text { le }\end{array}$ & 1326.5 & 71.4 & 3775.5 & $34 \quad 69.4$ \\
\hline $\begin{array}{l}\text { Desfavo } \\
\text { rable }\end{array}$ & 3673.5 & 1428.6 & 1224.5 & $15 \quad 30.6$ \\
\hline Total & 49100 & $49 \quad 100$ & 49100 & $49 \quad 100$ \\
\hline
\end{tabular}

La tabla 2, muestra que en las dimensiones: Información el $73.5 \%$ (36) tiene una percepción desfavorable y el $26.5 \%$ (13) es favorable, en la dimensión campo de presentación el $71.4 \%$ (35) manifiesta una percepción favorable y el 28.6\% (13) es desfavorable, en la dimensión actitud 75.5\% (37) posee una percepción favorable y el $24.5 \%$ (12) es desfavorable y por último en la dimensión religión el $69.4 \%$ (34) tiene una percepción favorable y el $30.6 \%$ (15) es desfavorable.

\section{DISCUSIÓN}

En la presente investigación se determinó la percepción de la imagen social del profesional de enfermería en pacientes del Puesto de Salud de Pedro Castro Alva, se encontró que el 54\% de los pacientes tiene una percepción favorable del profesional de enfermería y el $46 \%$ de ellos tiene una percepción desfavorable.

En cuanto a sus dimensiones la mayor parte de los pacientes indican que en la dimensión información el $73.5 \%$ tienen una percepción desfavorable de la imagen social de enfermería y el $26.5 \%$ favorable, esto se relaciona con la investigación de Avalos (2018) en la cual en sus resultados enuncia que el 99\% de las personas encuestadas afirman actividades técnicas como la toma de signos vitales y el cuidado de enfermos, olvidándose de otras áreas importantes como menciona Avalos, M. (2018) en sus resultados, que las personas no reconocen a los enfermeros en áreas como trabajos administrativos, educación nutricional, consejería, promoción de salud, detección de enfermedades, planificación familiar, prevención de riesgos, docencia e investigación, no son reconocidos por gran parte de la población.

Esto también se relaciona con los estudios de Muñoz \& Consuegra (2015) y Valencia \& Pacheco (2018) en la que sus resultados presentan las funciones de enfermería como actividades técnicas como: inyectar, ayudante del médico y en un menor porcentaje prevenir y recetar.

Loza (2019) y Avalos, M. (2018) encontraron que el profesional de enfermería no es independiente en sus funciones, aun se puede apreciar el estereotipo de que el profesional es ayudante del médico o muchas de ellas relacionándolas a tareas prescritas por estos, aun la población no comprende que se realiza el trabajo delegado por otros profesionales, olvidándose por completo que el profesional de enfermería muchas veces toma la decisión para el mejoramiento de su salud.

En cuanto a esta dimensión menciona al individuo y como aprecia las áreas, funciones y desarrollo del profesional en la sociedad, esto quiere decir que gran parte de la población aún desconoce y tiene poco conocimientos acerca de la profesión de enfermería, muchas de ellas asociándolo a estereotipos e imágenes de su rol que en su pasado estuvo desarrollado, partiendo de la idea que la imagen social es identidad los sujetos involucrados son los responsables del mismo constructo de estas percepciones, como menciona Heirle (2009) que no solo cambiar las formas, es decir los uniformes, los signos, los títulos, los niveles académico, sin comprender, y sobre todo, sin explicar a los medios la estructura que determina dichos cambios, no condiciona el cambio que percibe la sociedad, porque para ella, la imagen sigue teniendo la misma naturaleza.

Por otro lado, en la dimensión, campo de presentación, el $73 \%$ de los pacientes encuestados posee una percepción favorable de la imagen social de enfermería y el $27 \%$ es desfavorable esto pone en evidencia que el cuidado que realiza y la respuesta del paciente son objetivos, este a la misma ves tiene similitudes con el estudio de Avalos (2018) en la que en sus resultados menciona que $96 \%$ de la población reconoce que el accionar de enfermería le proporciona mejoría en su salud, también se relaciona con el estudio de Ramos (2015) en la que menciona 
en sus resultados que el $80,9 \%$ de los usuarios afirma que las enfermeras están preparadas para la identificación de necesidades.

En esta dimensión nos hace mención la respuesta del sujeto frente al cuidado y su interrelación, en la cual se puede apreciar de manera favorable en el estudio de Mauricio, \& Janjachi (2017), que encontró que los valores que resaltan en el profesional de enfermería es la confianza, seguridad y respeto.

En la dimensión actitud el 75.5\% de los pacientes encuestados tiene una percepción favorable, sabiendo que en esta dimensión recalca el modo de actuación de enfermería en el desarrollo del cuidado, encontrándose parecidos resultados con los de Mauricio, \& Janjachi (2017) en la que menciona una impresión buena respecto, a la actitud, en la que está conformada por el tono de voz, el trato, el uniforme, la explicación de dudas que se le realiza hacia el paciente desde el momento de la atención es adecuada y por lo cual se reporta un mayor grado de bienestar, esto también difiere con el estudio de Duhovnik \& Moraga (2015) en su estudio en la que menciona que los factores que influyen para la conformación de la imagen social como el trato, la comunicación, el apoyo emocional, solución de dudas en inquietudes son percibidos de manera inadecuada.

En la dimensión religión, el $69.4 \%$ de los entrevistados tienen una percepción favorable, esto tiene semejanzas con el estudio de Loza (2019), que afirma que la religión es importante ya que es parte de su formación, esto también tiene parecido con el estudio de Mauricio \& Janjachi (2017) en la cual se encontró que el aspecto espiritual es importante en el cuidado de la persona.

La religión en enfermería es considerada parte de la profesión ya que históricamente esta se relacionaba a la religión y eran las mujeres que se encargaban del cuidado de las personas más enfermas y esto era visto como ayuda al prójimo.

\section{CONCLUSIONES}

La percepción de la imagen social del profesional de enfermería para los pacientes del Puesto de Salud pedro Castro Alva es favorable.

El $54 \%$ de los pacientes poseen una percepción favorable de la imagen social del profesional de enfermería y el $46 \%$ desfavorable.

En la dimensión información el 73.5\% tiene una percepción desfavorable y el 26.5\% (13) favorable, en la dimensión campo de presentación el $71.4 \%$ manifiesta una percepción favorable y el $28.6 \%$ desfavorable, en la dimensión actitud $75.5 \%$ posee una percepción favorable y el $24.5 \%$ desfavorable y en la dimensión religión el $69.4 \%$ tiene una percepción favorable y el 30.6\% (15) desfavorable.

\section{REFERENCIAS BIBLIOGRÁFICAS}

Avalos, M. (2018). Imagen social del profesional de enfermería desde la perspectiva del trabajador en empresa privada y pública en la provincia de san José, II cuatrimestre 2018. (Trabajo para optar el título de enfermera) .Disponibleenhttp://webcache.googleusercont ent.com/search?q=cache:tfcD1cG64ksJ:13.6 5.82.242:8080/xmlui/bitstream/handle/cenit/ 3407/ENFE387.pdf\%3Fsequence\%3D1+\&cd $=1 \& \mathrm{hl}=\mathrm{es} \& \mathrm{ct}=\mathrm{clnk} \& \mathrm{gl}=\mathrm{pe}$

Costana, E. (2014). Imagen social de la enfermería. Estrategias para la reafirmación social de la profesión. (Trabajo fin de grado). Valladolid. Universidad de Valladolid. Disponibleen. http://uvaduc.uva.es/handle/10324/5101

Duhovnik, A., Moraga, J. (2015). Imagen social y profesional de la enfermera desde la percepción de pacientes hospitalizados. Disponible en https://bdigital.uncu.edu.ar/ob j etos_digitales/8618/moraga-jesica.pdf.

Errasti B., Arantzamendi M., Canga N. (2012). La imagen social de la enfermería:una profesión a conocer. Anales del Sistema Sanitario de Navarra, 35(2), 269-283.https://dx.doi .org/10.4321/S1137-66272012000200009.

Heierle C. (2009). La imagen de la enfermera a través de los medios de comunicación: la prensa escrita. Index de enfermería. 18(2), 95 $-98$

Loza, M. (2018). Percepción de los usuarios sobre la imagen que proyecta el profesional de enfermería en un establecimiento de primer nivel de atención de Lima 2018. (Tesis para optar el Título Profesional de Licenciada en Enfermería). Universidad Nacional Mayor de San Marcos.

Mauricio, C., Janjachi, B. (2017). Imagen social del profesional de Enfermería según la percepción de los pacientes del Hospital de Huaycan 2016.

Mieles, S. Parreño, D. (2017). Estudio de la percepción sobre la imagen social del rol de enfermería en la ciudad de santo domingo de los Tsáchilas periodo diciembre 2017. Pontificia Universidad Católica del Ecuador.

Muñoz F. (2007). Imagen de la enfermería en los medios de comunicación: reflejo de una realidad social. Revista electrónica internacional de cuidados. 7(2), 32-37. 
OMS. (2020). El 2020 será el año de la enfermería. Disponible en: https://www.enferm eria21. com/diario-dicen/el-2020-sera-el-ano-delaenfermeria/RAE. (2020). Enfermería.Dispo nible en: https://dle.rae.es/enfermer $\% \mathrm{C} 3 \% \mathrm{ADa}$

Ramos, S., Brito, P., Sánchez, M., Gutiérrez, D., (2015). Percepción de los pacientes acerca de la profesión enfermera en atención primaria. Ene, 9(3)

Supo, J. (2015). Seminarios de Investigación científica. Jalisco: Adventure. Perú.

Valencia, J., Pacheco, J. (2018). Imagen social del profesional de enfermería según la percepción del paciente hospitalizado en el servicio de cirugía del Hospital Regional cusco 2018. 